\title{
Strigiformes e Caprimulgiformes em Santa Catarina, sul do Brasil: Registros relevantes e novas localidades
}

\author{
Evair Legal* \\ Tiago João Cadorin \\ Glauco Ubiratan Kohler \\ Laboratório de Ecologia e Ornitologia, Instituto de Pesquisas Ambientais \\ Universidade Regional de Blumenau - FURB \\ Rua Antônio da Veiga, 140, CEP 89012-900, Blumenau - SC, Brasil \\ *Autor para correspondência \\ evairlegal@yahoo.com.br
}

Submetido em 05/02/2009

Aceito para publicação em 21/08/2009

\section{Resumo}

Com as crescentes pesquisas ornitológicas em Santa Catarina, muitas localidades já possuem satisfatório conhecimento sobre sua avifauna, porém, alguns táxons ainda são subamostrados na maioria desses estudos avifaunísticos. Tal subamostragem de certos grupos de aves, como as noturnas (Strigiformes e Caprimulgiformes), pode prejudicar a avaliação do status de ocorrência e situação populacional dessas espécies. Neste sentido, apresentamos registros de sete espécies de Strigiformes (Megascops sanctaecatarinae, Pulsatrix koeniswaldiana, Strix hylophila, S. virgata, Glaucidium minutissimum, G. brasilianum e Asio stygius) e seis de Caprimulgiformes (Nyctibius griseus, Podager nacunda, Nyctidromus albicollis, Caprimulgus sericocaudatus, C. longirostris e Macropsalis forcipata) observados recentemente em nossos estudos, sendo que todas as espécies aqui apresentadas foram registradas em ao menos uma localidade inédita para Santa Catarina.

Unitermos: Aves noturnas, Caprimulgiformes, distribuição, Santa Catarina, Strigiformes

\section{Abstract}

Strigiformes and Caprimulgiformes in Santa Catarina State, southern Brazil: Relevant records and new localities. With the growing ornithological research in Santa Catarina, many localities have already acquired good knowledge about their avifauna, but, some groups of birds are still sub-sampled in most of these ornithological studies. This sub-sampling of certain avian groups, such as the nocturnal (Strigiformes and Caprimulgiformes), may prejudice the evaluation of occurrence status and population state of these species. Thus, we present records of seven Strigiformes species (Megascops sanctaecatarinae, Pulsatrix koeniswaldiana, Strix hylophila, S. virgata, Glaucidium minutissimum, G. brasilianum and Asio stygius) and six Caprimulgiformes (Nyctibius griseus, Podager nacunda, Nyctidromus albicollis, Caprimulgus sericocaudatus, C. longirostris and Macropsalis forcipata) observed in our studies, and all the species presented here were recorded in at least one unprecedented location in Santa Catarina.

Key words: Caprimulgiformes, distribution, nocturnal birds, Santa Catarina, Strigiformes 


\section{Introdução}

Após a publicação, em 1996, da mais importante obra sobre as aves de Santa Catarina (Rosário, 1996), a qual abrangia todas as informações até então conhecidas sobre a avifauna do Estado, houve uma ampliação do conhecimento sobre esse grupo, principalmente envolvendo distribuição geográfica, porém, poucos desses trabalhos incluem dados acerca da avifauna noturna. No Estado eram conhecidas 16 espécies pertencentes à ordem Strigiformes e 11 espécies à ordem Caprimulgiformes (Rosário, 1996). A grande maioria destas espécies era tida como rara e algumas sequer possuíam localidade de registro especifica conhecida, sendo sua ocorrência em Santa Catarina inferida a partir de citações bibliográficas de cunho genérico (Rosário, 1996). Hoje se sabe que, para muitas espécies, isso se deveu à escassez de estudos da época e ao fato de que certas regiões foram insuficientemente amostradas, sendo que algumas nunca haviam sido pesquisadas. Alguns estudos recentes (e.g. Piacentini et al., 2006; Accordi e Barcellos, 2008; Rupp et al., 2008) acrescentaram registros importantes para a avifauna de Santa Catarina (inclusive uma espécie noturna inédita), assim como localidades não mencionadas anteriormente na literatura. Apresentamos a seguir informações sobre registros de 13 espécies de aves noturnas, sete Strigiformes e seis Caprimulgiformes, obtidos metódica e esporadicamente em diferentes áreas, sendo que todas as espécies relatadas tiveram ao menos um registro em uma localidade inédita para Santa Catarina.

\section{Material e Métodos}

\section{Área de estudo}

Os dados apresentados foram obtidos entre setembro de 2004 e março de 2009, em áreas de Floresta Ombrófila Densa do Vale do Itajaí e Vale do Tijucas, e em área de Floresta Ombrófila Mista no município de Abelardo Luz, oeste de Santa Catarina.

As localidades com maior número de registros são apresentadas abaixo e as demais esquematicamente no Apêndice 1, sendo classificadas com acrônimos, os quais serão utilizados na descrição dos registros das espécies.
PNSI - Parque Nacional da Serra do Itajaí: área com 57.374 ha distribuídos em nove municípios (Apiúna, Ascurra, Blumenau, Botuverá, Gaspar, Guabiruba, Indaial, Presidente Nereu e Vidal Ramos), composta por Floresta Ombrófila Densa Submontana, Montana e Altomontana. A altitude do PNSI varia entre 80 e $1.039 \mathrm{~m}$. Os registros apresentados deram-se nas seguintes regiões: PNSI1: Segunda Vargem, município de Blumenau: área composta por vegetação em estádio inicial e avançado de regeneração. Possui alguns ribeirões e lagoas artificiais; PNSI2: Vale do Espingarda, município de Indaial: grande parte composta por vegetação secundária em estádio médio de regeneração; PNSI3: Terceira Vargem, município de Blumenau: possui floresta secundária em avançado estádio de regeneração e pequenos cursos d'água, próximos à uma área de floresta primária.

RCE - RPPN Chácara Edith, município de Brusque: área com 415,79 ha de Floresta Ombrófila Densa Submontana, a qual com os arredores formam um fragmento florestal de aproximadamente 1,2 mil ha, grande parte composta por floresta secundária em médio e avançado estádio de regeneração. Em 2006, a RCE foi reconhecida como Posto Avançado da Biosfera da Mata Atlântica, primeiro do segmento no Estado de Santa Catarina.

\section{Metodologia}

Entre as localidades aqui reportadas, apenas na RCE foi empregado estudo sistemático envolvendo a avifauna noturna, a qual foi desenvolvida com amostragem em duas noites mensais durante o período da lua cheia e crescente, entre novembro de 2006 e julho de 2007.

Para isso, foram demarcados 11 pontos fixos, distantes 200m entre si, englobando áreas abertas, bordas e interior de floresta. Em cada ponto foi reproduzida a vocalização das espécies com auxilio de playback em sequência pré-estabelecida.

Nas demais localidades os contatos deram-se de forma espontânea durante estudos esporádicos com avifauna.

A identificação das espécies se deu através de contatos visuais (por observações diretas) ou auditivos 
(através de cantos, pios, chamados e gritos de alerta), e quando possível tendo documentação através de fotografia (apresentadas no texto referente à espécie) ou gravação das vocalizações, das quais algumas foram indexadas no arquivo sonoro digital Xeno-canto (XC; www.xeno-canto.org).

A nomenclatura científica e ordem sistemática seguem a Lista das Aves do Brasil, disponibilizada pelo Comitê Brasileiro de Registros Ornitológicos (CBRO, 2008).

\section{Resultados e Discussão}

\section{Megascops sanctaecatarinae (corujinha-do-sul)}

$\mathrm{Na}$ RCE, foi ouvida nos dias 5 (dois indivíduos) e 8 de agosto, 30 de setembro, 2 e 8 de novembro de 2006; 19 de abril de 2008 (registro fotográfico). A espécie pôde ser encontrada tanto na área de capoeirão nas partes mais periféricas da RCE como na área mais interna, com vegetação secundária em avançado estádio de regeneração. Gravada no BAC, em ambiente de capoeirão, no dia 17 de outubro de 2006. Ouvida no PNMSFA, região central do município de Blumenau, em 15 de agosto de 2007. Estes registros apontam novas localidades de ocorrência desta espécie, bem como as primeiras citações para o Vale do Itajaí. Rosário (1996) cita um único registro da espécie, tratada então como Otus atricapilla, para Santa Catarina, realizado no município de Joinville, em 1904. Accordi e Barcellos (2008) supõem que esta espécie seja subestimada em Santa Catarina, visto que no Rio Grande do Sul é comum em toda a parte norte do Estado. Estes autores apresentam dois registros no vale do rio Uruguai, realizados nos municípios de Zortéa e Piratuba.

Pulsatrix koeniswaldiana (murucututu-de-barrigaamarela)

Na FURBCI, área urbana do município de Blumenau, pôde ser observada no período diurno do dia 21 de junho de 2006. Em Blumenau, um indivíduo foi encontrado morto em 11 de julho de 2005 (depositado na Coleção Zoológica da Universidade Regional de Blumenau (CZFURB; FURB 10617) e outro em 2 de julho de 2008. Um jovem, atraído por ovos e pedaços de frango, foi fotografado na RCE por W. Moreli na borda da floresta, em 12 de janeiro de 2007. Nesta mesma Unidade de Conservação (UC) foi ouvida no dia 19 de abril de 2008. No PNSI3 foram obtidos registros em 23 de março (um indivíduo em voo segurando um roedor nas patas), 27 de julho (um indivíduo observado e ouvido) e 17 de agosto de 2007 (fotografado). No dia 28 de julho de 2008, um indivíduo macho foi encontrado morto, abaixo da rede elétrica no BPM, posteriormente depositado na CZFURB (FURB 13413; Figura 1). Em 3 de agosto de 2008 um indivíduo foi ouvido em VRC. Dois indivíduos ouvidos e um fotografado (Figura 2) em 3 de outubro de 2008 no EA. Na ocasião também pôde ser observado a predação de um anfíbio (Hypsiboas faber) por esta espécie. Os registros de Brusque, Indaial e Vidal Ramos representam áreas de ocorrência da espécie ainda não citadas para Santa Catarina. Considerada rara no Estado por Rosário (1996), eram conhecidos apenas registros para o Parque Estadual da Serra do Tabuleiro (PEST), em Santo Amaro da Imperatriz e outro no Parque Ecológico Artex (hoje inserido no PNSI) em Blumenau. Piacentini et al. (2006) mencionam registros para Joinville, Ilhota e São Domingos. Accordi e Barcellos (2008) registraram a espécie no município de Piratuba. Bencke e Bencke (1999a; 1999b) relatam a existência de dois indivíduos provenientes de São Cristóvão do Sul e um de Lontras, sendo todos depositados na coleção da Pontifícia Universidade Católica do Rio Grande do Sul (PUCRS), Porto Alegre, Rio Grande do Sul. Para o centro-oeste de Santa Catarina, Favretto et al. (2008), mencionam a espécie no Parque Natural Municipal do Rio do Peixe (PMNRP), município de Joaçaba.

\section{Strix hylophila (coruja-listrada)}

Vocalização gravada: AL, em setembro de 2006; VRC, em 1 de abril de 2007, próximo a um lago; MC, em 15 de agosto de 2008. Abelardo Luz e Vidal Ramos não possuíam ocorrência mencionada para a espécie no Estado. Rosário (1996) cita sua ocorrência em São Bento do Sul, Calmon, Florianópolis, Nova Trento/Botuverá e Rio dos Cedros. Para Ilha de Santa Catarina, há registros na Costa de Dentro e em diversos outros locais, sendo que esta espécie é, aparentemente, a coruja noturna localmente mais comum (Naka et al., 2000; Naka e Rodrigues, 2000). Piacentini et al. (2006) registraram a coruja-listrada em São Domingos, Nova Veneza e Curitibanos. Há também 


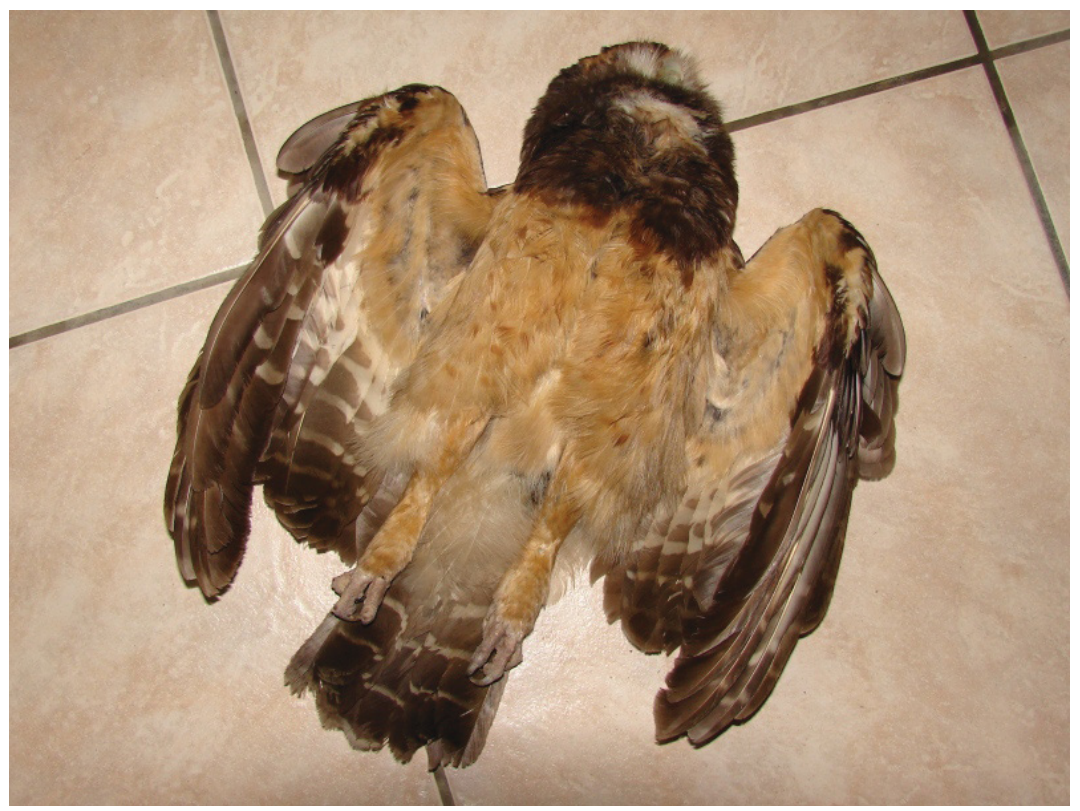

FIGURA 1: Exemplar de Pulsatrix koeniswaldiana encontrado no dia 28 de julho de 2008 em Brusque, SC, antes da preparação e depósito na Coleção Zoológica da Universidade Regional de Blumenau (FURB 13413).

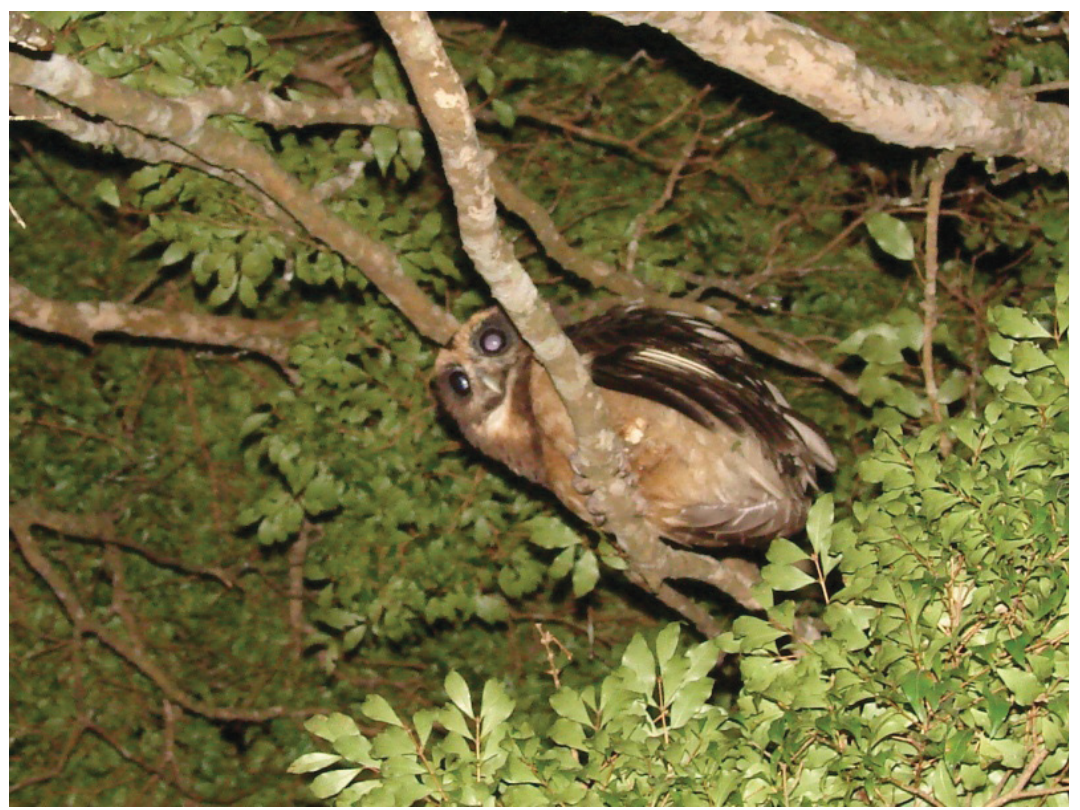

FIGURA 2: Indivíduo de Pulsatrix koeniswaldiana fotografado no dia 3 de outubro de 2008 na localidade Encano Alto, Indaial, SC.

registros em Piratuba e Joaçaba, região oeste (Accordi e Barcellos, 2008; Favretto et al., 2008). Esta espécie é endêmica da Floresta Atlântica e considerada globalmente quase-ameaçada de extinção (categoria Near Threatened) (BirdLife International, 2008).

\section{Strix virgata (coruja-do-mato)}

Um indivíduo morto (provavelmente colidiu com a fiação da rede elétrica, visto que estava logo abaixo da mesma) encontrado no BAC em 7 de setembro de 2004. Outros registros foram obtidos na RCE em 2 e 7 de 
novembro de 2006. Em 23 de maio de 2006 um indivíduo teve sua vocalização gravada no PNSI3. Estes registros realizados em Brusque e Indaial adicionam novas localidades de ocorrência da espécie em Santa Catarina. Bencke e Bencke (1999b) a documentaram pela primeira vez no Estado, através de um espécime procedente de Massaranduba, o qual está depositado na coleção da PUCRS. Porém, assim como Rosário (1996), Bencke e Bencke (1999b) desconheciam uma pele procedente de Joinville, depositada no Field Museum of Natural History (FMNH), em Chicago, Estados Unidos. Laps et al. (2004) relatam a existência desta pele no FMNH e outras quatro na CZFURB, todas de Blumenau.

\section{Glaucidium minutissimum (caburé-miudinho)}

Na RCE, registros em 10 de junho, 10 de julho (gravação), 14 e 30 de setembro, 13 de outubro, 2 e 7 de novembro de 2006, 15 de julho, 7 de setembro (dois indivíduos) e 7 de novembro de 2007, 20 de fevereiro (vocalização gravada) e 19 de abril de 2008. Em 30 de julho de 2008, dois indivíduos continuavam a vocalizar mesmo com o comportamento mobbing de outras aves (e. g. Euphonia violacea, Trichothraupis melanops, Tangara cyanocephala e Thalurania glaucopis), ou seja, uma associação agressiva de espécies na tentativa de rechaçar este predador e alertar outras aves sobre sua presença (Sigrist, 2006). Registros realizados no PNSI3, em 2 de junho e 27 de julho de 2007 (dois indivíduos ouvidos e observados), e em 8 de novembro de 2008 (um indivíduo observado). $\mathrm{Na} \mathrm{RB}$, um indivíduo teve sua vocalização gravada em 9 de maio de 2006. Quando presente na área, a espécie respondia prontamente à reprodução do seu canto, mesmo quando este era assoviado. Estas são novas localidades de ocorrência de G. minutissimum, bem como a segunda citação da espécie para Santa Catarina, além dos registros mais meridionais conhecidos no Estado. O primeiro registro foi obtido em 1994 no Parque Botânico Morro do Baú (Rosário, 1996). König e Weick (2005), convencidos de que $G$. mooreorum seria um sinônimo de G. minutissimum e que a verdadeira $G$. minutissimum estaria limitada ao nordeste do Brasil, nomearam as populações do sudeste, sul do Brasil e Paraguai oriental como uma espécie distinta: G. sicki, cujo tipo depositado no Staatliches Museum für Naturkunde Stuttgart (SMNS), Alemanha, é proveniente de Santa Catarina, sem data e localidade específica. No entanto, G. mooreorum continua sendo tratado como nome válido (CBRO, 2008) e G. sicki, inválido, por se tratar de sinonímia de G. minutissimum (V. Q. Piacentini, comunicação pessoal).

\section{Glaucidium brasilianum (caburé)}

Em 17 de fevereiro de 2007, observada no período diurno pousada na fiação elétrica em PG. Ouvida em 15 de novembro de 2007, no PNSI3, ocorrendo ao lado da espécie anterior. Estes municípios não possuem ocorrência mencionada na literatura para a espécie, sendo conhecidos registros apenas para Mondaí, Bom Retiro, Santo Amaro da Imperatriz, Nova Trento/ Botuverá, Garuva e Corupá (Rosário, 1996).

Asio stygius (mocho-diabo)

Registros auditivos na RCE: 30 de setembro e 7 de novembro de 2006, 31 de janeiro, 27 e 28 de fevereiro, 9 e 30 de março, 15 de abril e 27 de novembro de 2007. Em 2 de fevereiro de 2007 foi visualizada após playback. Em 15 de agosto de 2007, ouvida no PNMSFA. Ouvido e visualizado no PNSI2, em 20 de outubro de 2006 e 2 de fevereiro de 2007. Os municípios de Brusque e Indaial representam novas localidades para a espécie. No Estado, com registros provavelmente para o município de Lontras e PEST, era considerada rara por Rosário (1996). Acredita-se que esta espécie esteja sendo subamostrada no Vale do Itajaí, visto que não foi observada em estudos anteriores realizados nesta região (e. g. Zimmermann 1992; 1993; 1995; 1999; Marterer, 1996). Para a Ilha de Santa Catarina, Naka e Rodrigues (2000) a consideram rara, com um único registro. Na CZFURB há três peles provenientes de Blumenau (Laps et al. 2004).

Nyctibius griseus (mãe-da-lua)

Ouvida na RCE em 13 e 28 de outubro (XC 15936), 2 e 7 de novembro (dois indivíduos) e 15 de dezembro de 2006, 7 de janeiro (Figura 3) e 2 de fevereiro e 9 de março de 2009. Em 2 de fevereiro de 2007, dois indivíduos foram observados a poucos metros atraídos por playback. Um indivíduo foi ouvido no amanhecer de 25 de novembro de 2006 no PNSI1, em uma área de capoeirão. Registros da espécie já eram conhecidos para Blumenau, porém a menção da ocorrência da espécie para Brusque é inédita. São conhecidos ainda registros 


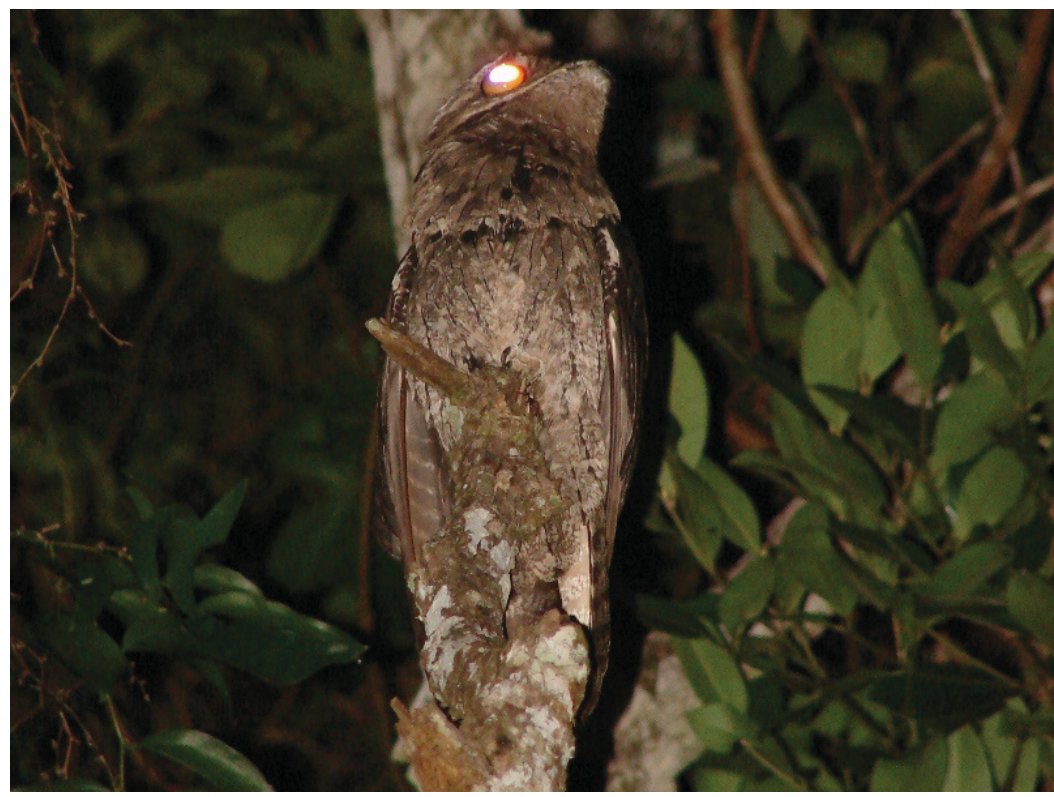

FIGURA 3: Exemplar de Nyctibius griseus fotografado na RPPN Chácara Edith, Brusque, SC, no dia 7 de janeiro de 2009.

para Lontras, Camboriú, Florianópolis, Itapoá e Doutor Pedrinho (Rosário, 1996; Naka et al., 2000; Naka e Rodrigues, 2000; Rupp et al., 2008).

\section{Podager nacunda (corucão)}

Dois indivíduos foram observados em voo em GAC no dia 30 de maio de 2008. Em Santa Catarina há registros anteriores para Blumenau, Corupá, Florianópolis, Imbituba, Itaiópolis e São Joaquim, muitos realizados em áreas urbanas (Rosário, 1996; Naka et al., 2000; Naka e Rodrigues, 2000; Amorim e Piacentini, 2006; Rupp et al., 2008).

\section{Nyctidromus albicollis (bacurau)}

Em 15 de setembro de 2008, ouvida no BAC e em 22 de fevereiro de 2009 na RCE (gravada). Eram conhecidos apenas registros em Blumenau, Doutor Pedrinho, Ilha de Santa Catarina, PMNRP, Rio dos Cedros e São Francisco do Sul (Rosário, 1996; Naka et al., 2001; Favretto et al., 2008; Azevedo, 2006).

Caprimulgus sericocaudatus (bacurau-rabo-deseda)

$\mathrm{Na}$ RCE, um indivíduo foi ouvido em $15 \mathrm{de}$ dezembro de 2006 (vocalização gravada), 7 de novembro, 30 de março de 2007, 24 de fevereiro (gravação) e 14 de março de 2009 (XC 31200), neste último, quando um indivíduo pôde ser visualizado empoleirado em um galho de baixa altura, conforme observado por Sick (1997). Em 20 de outubro de 2006, dois indivíduos ouvidos durante o crepúsculo na periferia do PNSI2. Em 03 de agosto de 2008, ouvida ao amanhecer do dia, na VRC. Apenas recentemente C. sericocaudatus foi reportado em Santa Catarina, nos municípios de Blumenau, Gaspar, Presidente Nereu, Indaial e Apiúna (Rupp et al., 2008).

\section{Caprimulgus longirostris (bacurau-da-telha)}

No dia 1 de março de 2007, ouvida no BAC, próximo ao telhado de uma residência. Esta espécie é conhecida pela utilização de telhados como local de abrigo além de aproveitar a iluminação elétrica para a captura de insetos (Sick, 1997). Rupp et al. (2008) registraram esta espécie através de gravação da vocalização em Doutor Pedrinho e através de uma pele proveniente de uma área urbana de Blumenau, a qual se encontra depositada na CZFURB (FURB 13231). Existem três registros anteriores a estes, um realizado no Campo dos Padres, município de Bom Retiro, e dois outros em Florianópolis (Rosário, 1996; Naka e Rodrigues, 2000). 


\section{Macropsalis forcipata (bacurau-tesoura-gigante)}

Um indivíduo foi observado em VRPS em 3 de abril de 2007, pousado em uma estrada de terra. Era conhecido, segundo Rosário (1996), um registro considerado provável para Brusque e outro em Camboriú. Rupp et al. (2008) mencionam a ocorrência desta espécie no município de Doutor Pedrinho. Accordi e Barcellos (2008) citam um indivíduo macho, sem data nem local de procedência, depositado na coleção do Colégio Pedro II, Blumenau, e registro em Piratuba.

Os registros aqui apresentados refletem a evidente necessidade de mais estudos ornitológicos em Santa Catarina, notadamente com as aves noturnas, a fim de tornar mais precisas e confiáveis as avaliações da situação populacional de cada espécie no Estado e aspectos da conservação.

\section{Agradecimentos}

Agradecemos ao Sr. Wilson Moreli e à Sra. Lígia Moreli pelo apoio fornecido às pesquisas realizadas na RPPN Chácara Edith, ao Departamento de Ciências Naturais da Universidade Regional de Blumenau por ceder transporte ao PNSI, ao Vítor de Queiroz Piacentini pelas informações e comentários, ao Rony Paolin Hasckel pela companhia em campo e aos revisores deste manuscrito pelas correções e sugestões.

\section{Referências}

Accordi, I. A.; Barcellos, A. 2008. Novas ocorrências e registros notáveis sobre distribuição de aves em Santa Catarina, sul do Brasil. Biotemas, 21 (1): 85-93.

Amorim, J. F.; Piacentini, V. de Q. 2006. Novos registros de aves raras em Santa Catarina, Sul do Brasil, incluindo os primeiros registros documentados de algumas espécie para o Estado. Revista Brasileira de Ornitologia, 14 (2): 145-149.

Azevedo, M.A. G. 2006. Contribuição de estudos para licenciamento ambiental aoconhecimento da avifauna de Santa Catarina, Sul do Brasil. Biotemas, 19 (1): 93-106.

Bencke, G. A.; Bencke, C. S. C. 1999a. The potential importance of road deaths as a cause of mortality for large forest owls in southern Brazil. Cotinga (Sandy), 11 (1): 79-80.

Bencke, G. A.; Bencke, C. S. C. 1999b. More road-killed owls and a new record or Santa Catarina, Brazil. Cotinga (Sandy), 11 (1): 69.
BirdLife International. 2008. Species factsheet - Strix hylophila. Disponível em $<$ http://www.birdlife.org $>$. Acesso em 02 de agosto de 2008.

CBRO - Comitê Brasileiro de Registros Ornitológicos. 2008. Listas das aves do Brasil. Versão 5/10/2008. Disponível em <http://www. cbro.org.br>. Acesso em 08 de maio de 2009.

Favretto, M. A.; Zago, T.; Guzzi, A. 2008. Avifauna do Parque Natural Municipal Rio do Peixe, Santa Catarina, Brasil. Atualidades Ornitológicas, 141: 87-93.

König, K. e Weick, F. 2005. Ein neuer Sperlingskauz (Aves: Strigidae) aus Südostbrasilien. Stuttgarter Beitr. Stuttgarter Beiträge zur Naturkunde, Serie A, 688: 1-12.

Laps, R. R.; Borchardt-Jr, C.A.;Alves, E. W.;Althoff, S. L.;Azevedo, M. A. G. 2004. Registro de aves raras ou pouco conhecidas para o Estado de Santa Catarina. Anais do XII Congresso Brasileiro de Ornitologia, Blumenau, Brasil, p.258.

Marterer, B. T. P. 1996. Avifauna do Parque Botânico do Morro do Baú. Riqueza, aspectos de frequência e abundância. FATMA, Florianópolis, Brasil, 74pp.

Naka, L. N.; Rodrigues, M. 2000. As Aves da Ilha de Santa Catarina. Editora da Universidade Federal de Santa Catarina, Florianópolis, Brasil, 294pp.

Naka, L. N.; Mazar Barnett, J.; Kirwan, G. M.; Tobias, J. A.; Azevedo, M. 2000. New and noteworthy birds records from Santa Catarina State, Brazil. Bulletin of the British Ornithologists' Club, 120: 237-250.

Naka, L. N.; Mazar Barnett, J.; Kirwan, G. M.; Tobias, J. A.; Buzzetti, D. 2001. Records of bird species previously considered uncommon in Santa Catarina state, Brazil. Cotinga, 16: 68-70.

Piacentini, V. de Q.; Ghizoni-Jr, I. R.; Azevedo, M. A. G.; Kirwan, G. M. 2006. Sobre a distribuição de aves em Santa Catarina, Brasil, parte I: registros relevantes para o Estado ou inéditos para a Ilha de Santa Catarina. Cotinga, 26: 25-31.

Rosário, L. A. 1996. As aves de Santa Catarina: Distribuição geográfica e meio ambiente. FATMA, Florianópolis, Brasil, 326pp.

Rupp, A. E.; Brandt, C. S.; Fink, D.; Silva, G. T.; Laps, R. R.; Zimmermann, C. E. 2008. Registros de Caprimulgiformes e a primeira ocorrência de Caprimulgus sericocaudatus (bacurau-rabode-seda) no Estado de Santa Catarina, Brasil. Revista Brasileira de Ornitologia 15(4): 605-608.

Sick, H. 1997. Ornitologia brasileira. Nova Fronteira, Rio de Janeiro, Brasil, 823pp.

Sigrist, T. 2006. Aves do Brasil. Uma visão artística. Birds of Brazil. An artistic view. Fosfertil, São Paulo, Brasil, 672pp.

Zimmermann, C. E. 1992. Uma contribuição à ornitologia catarinense - Levantamento preliminar da ornitofauna do Parque Ecológico Artex. Dynamis, 1 (1): 69-80.

Zimmermann, C. E. 1993. Nota sobre a avifauna do Parque Ecológico Spitzkopf - Blumenau/SC. Dynamis, 1 (3): 7-13.

Zimmermann, C. E. 1995. Novas informações sobre a avifauna do Parque Ecológico Artex. Biotemas, 8 (1): 7-20.

Zimmermann, C. E. 1999. Avifauna de um fragmento de Floresta Atlântica em Blumenau, Santa Catarina. Revista de Estudos Ambientais, 1 (3): 101-112. 


\section{APÊNDICE 1:}

Dados gerais das localidades de registros apresentados no trabalho.

\begin{tabular}{ccccc}
\hline $\begin{array}{c}\text { Área de } \\
\text { estudo }\end{array}$ & Município & Localidade & $\begin{array}{c}\text { Coordenadas Geo- } \\
\text { gráficas }\end{array}$ & $\begin{array}{c}\text { Altitude } \\
(\mathbf{m})\end{array}$ \\
\hline AL & Abelardo Luz & Próximo ao Rio Chapecó & $26^{\circ} 34^{\prime} \mathrm{S}-52^{\circ} 24^{\prime} \mathrm{W}$ & 730 \\
\hline BAC & Brusque & Bairro Águas Claras & $27^{\circ} 08^{\prime} \mathrm{S}-48^{\circ} 54^{\prime} \mathrm{W}$ & 50 \\
\hline BPM & Brusque & Bairro Primeiro de Maio & $27^{\circ} 07^{\prime} \mathrm{S}-48^{\circ} 54^{\prime} \mathrm{W}$ & 36 \\
\hline EA & Indaial & Encano Alto & $26^{\circ} 59^{\prime} \mathrm{S}-49^{\circ} 11^{\prime} \mathrm{W}$ & 184 \\
\hline FURBCI & Blumenau & Campus I - Universidade Regional de Blume- & $26^{\circ} 54^{\prime} \mathrm{S}-49^{\circ} 04^{\prime} \mathrm{W}$ & 36 \\
\hline GAC & Gaspar & nau (FURB) & $26^{\circ} 55^{\prime} \mathrm{S}-48^{\circ} 57^{\prime} \mathrm{W}$ & 10 \\
\hline MG & Indaial & Área central & $26^{\circ} 53^{\prime} \mathrm{S}-49^{\circ} 13^{\prime} \mathrm{W}$ & 150 \\
\hline MC & Nova Trento & Morro Geisler & $27^{\circ} 15^{\prime} \mathrm{S}-48^{\circ} 54^{\prime} \mathrm{W}$ & 520 \\
\hline PG & Presidente Getúlio & Morro da Cruz & $27^{\circ} 04^{\prime} \mathrm{S}-49^{\circ} 36^{\prime} \mathrm{W}$ & - \\
\hline PNMSFA & Blumenau & Parque Natural Municipal São Francisco de & $26^{\circ} 55^{\prime} \mathrm{S}-49^{\circ} 04^{\prime} \mathrm{W}$ & 70 \\
\hline PNSI1 & Blumenau & Parque Nacional da Serra do Itajaí & $27^{\circ} 03^{\prime} \mathrm{S}-49^{\circ} 05^{\prime} \mathrm{W}$ & 350 \\
\hline PNSI2 & Indaial & Parque Nacional da Serra do Itajaí & $27^{\circ} 01^{\prime} \mathrm{S}-49^{\circ} 08^{\prime} \mathrm{W}$ & 430 \\
\hline PNSI3 & Blumenau & Parque Nacional da Serra do Itajaí & $27^{\circ} 03^{\prime} \mathrm{S}-49^{\circ} 06^{\prime} \mathrm{W}$ & 380 \\
\hline RB & Blumenau & RPPN Bugerkopf & $27^{\circ} 00^{\prime} \mathrm{S}-49^{\circ} 04^{\prime} \mathrm{W}$ & 200 \\
\hline RCE & Brusque & RPPN Chácara Edith & $27^{\circ} 05^{\prime} \mathrm{S}-48^{\circ} 53^{\prime} \mathrm{W}$ & 36 \\
\hline VRPS & Vidal Ramos & Praça Stoltenberg & $27^{\circ} 20^{\prime} \mathrm{S}-49^{\circ} 19^{\prime} \mathrm{W}$ & 350 \\
\hline VRC & Vidal Ramos & Cinema & $27^{\circ} 18^{\prime} \mathrm{S}-49^{\circ} 13^{\prime} \mathrm{W}$ & 940 \\
\hline
\end{tabular}

